\title{
3D Printing Pneumatic Device Controls with Variable Activation Force Capabilities
}

\author{
Marynel Vázquez ${ }^{1,2}$, Eric Brockmeyer ${ }^{2}$, Ruta Desai ${ }^{1}$, \\ Chris Harrison ${ }^{2,3}$, Scott E. Hudson ${ }^{2,3}$

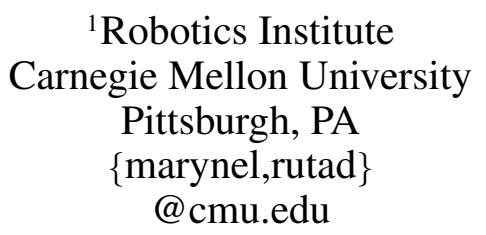 \\ ${ }^{2}$ Disney Research Pittsburgh \\ 4720 Forbes Avenue \\ Pittsburgh, PA \\ eric.brockmeyer \\ @ disneyresearch.com \\ ${ }^{3} \mathrm{HCI}$ Institute \\ Carnegie Mellon University \\ Pittsburgh, PA \\ \{chris.harrison, scott.hudson\} \\ @cs.cmu.edu
}

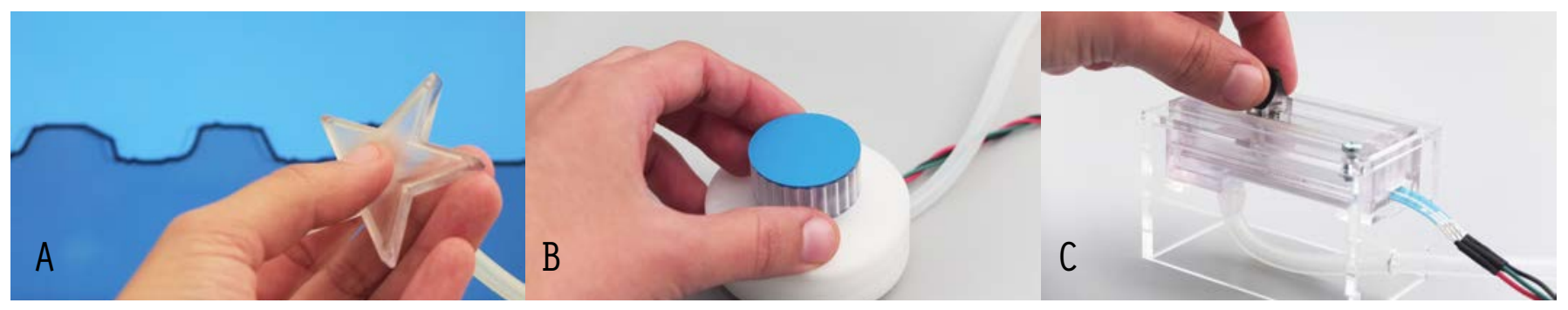

Figure 1. 3D printed pneumatic button (A), knob (B), and slider (C). We print these models with rigid and elastic materials, and in some cases add electronic components to the controls to sense user's input, such as potentiometers in the case of (B) and (C). Pressure sensing can be used to detect when users deform the elastic parts of the controls, like when they push the star. The graph behind this model shows the sensed pressure signal. We also use pressure to provide variable activation force capabilities.

\begin{abstract}
We explore 3D printing physical controls whose tactile response can be manipulated programmatically through pneumatic actuation. In particular, by manipulating the internal air pressure of various pneumatic elements, we can create mechanisms that require different levels of actuation force and can also change their shape. We introduce and discuss a series of example 3D printed pneumatic controls, which demonstrate the feasibility of our approach. This includes conventional controls, such as buttons, knobs and sliders, but also extends to domains such as toys and deformable interfaces. We describe the challenges that we faced and the methods that we used to overcome some of the limitations of current 3D printing technology. We conclude with example applications and thoughts on future avenues of research.
\end{abstract}

\section{Author Keywords}

3D printing; pneumatics; physical interfaces; haptics; rapid fabrication

\section{ACM Classification Keywords}

H.5.m. Information Interfaces and Presentation (e.g. HCI): Miscellaneous

Permission to make digital or hard copies of all or part of this work for personal or classroom use is granted without fee provided that copies are not made or distributed for profit or commercial advantage and that copies bear this notice and the full citation on the first page. Copyrights for components of this work owned by others than ACM must be honored. Abstracting with credit is permitted. To copy otherwise, or republish, to post on servers or to redistribute to lists, requires prior specific permission and/or a fee. Request permissions from permissions@ acm.org.

CHI'15, April 18 - 23, 2015, Seoul, Republic of Korea.

Copyright is held by the owner/author(s). Publication rights licensed to ACM.

ACM 978-1-4503-3145-6/15/04\$15.00

http://dx.doi.org/10.1145/2702123.2702569

\section{INTRODUCTION}

3D printing offers exciting new opportunities for the rapid creation of highly customized interactive components, such as pipe-based interactive objects [14] or optical elements [23]. In this work, we explore another class: a set of 3D printed physical controls whose feel and behavior can be adjusted dynamically. More specifically, we use pneumatics and standard sensing techniques for press, linear, and rotary input controls to create components with variable-activation force capabilities. These controls can be activated with light pressure, but require greater force in other circumstances. In this manner, users can receive tactile feedback through the controls based on the computational state of an interface, while providing discrete or continuous input. Additionally, some designs can be physically "highlighted" by raising them above a device surface. Illustrative examples are provided in Fig. 1.

The pneumatic controls proposed in this work are complementary to electro-mechanical variable activation force controls, such as the camera shutter of [12]. While more traditional mechanical controls may be appropriate for some applications, others may benefit from the compliance of pneumatic components. Pneumatics affords soft haptic feedback, which is harder to accomplish with mechanical actuation. In some cases it might also be easier and cheaper to route compressed air through complex geometries than to embed mechanical components inside a haptic control, such as a physical knob, button or slider. The benefits and limitations of pneumatic controls are further discussed in the paper.

We uncovered some interesting and unexpected design challenges with 3D printing technology for flexible components. 
In particular, current solutions for printing support material (sacrificial material designed to hold up part of the object while it is being printed, but removed later) proved problematical in several places. Even though we expect easier-toremove support material to become available for printing in the future, we describe current challenges and propose practical methods to mitigate them. This makes our controls easy to replicate, and facilitates their adoption and customization.

We envision that in the future we will be able to specify custom physical devices nearly as easily as we specify graphical user interfaces today - i.e., by picking components from a standardized library and editing them into a whole, functional unit. These devices could then be printed in their entirety or, in the nearer term, printed in parts and completed with simple assembly tasks. We move towards this end with the proposed, reusable set of proof of concept pneumatic components.

\section{RELATED WORK}

\section{Pneumatic Device Controls}

Harrison and Hudson used air chambers to create dynamic buttons that inflate or deflate on a visual display [6]. These chambers were manually constructed using clear acrylic, a masked glue layer, and a sheet of translucent latex. We build upon this work, and provide a more practical method for fabricating pneumatic buttons, as well as other device controls.

\section{D Printed Pneumatics}

Slyper et al. [17] explored pneumatically sensed interactive objects based on 3D printed hollow air chambers, similar to the ones that we fabricate. One of the main differences with our work, though, is that they used the chambers passively to prototype particular manipulation affordances on robot skins, rather than actively to provide haptic feedback or render computational states. The air chambers in [17] are soft and have a hole for support material removal. This aperture is also used to sense changes in internal air pressure, which occur when users manipulate the chambers, e.g., by twisting or stretching.

Other interesting pneumatic parts have been printed. For example, [4] fabricates pneumatic flowers with multi-material 3D printing. When pressure increases inside the flowers, their petals open, making it look like they blossom. While these flowers do not have electronic components in their interior, they seem to be fabricated in a similar fashion to our controls. Another example of 3D printed pneumatic parts is the peristaltic pump of [15]. This pump uses two integrated valves, a flexible silicone tube and an actuator to displace fluids. Hobbyists have also started to explore 3D printing pneumatic components, like a one way valve [22] or a pneumatic gripper that screws to the end of a standard plastic bottle [13].

Finally, Savage et al. [14] presented a tool for routing and optimizing pipes within 3D printed structures, which can support a range of pneumatic and other applications. These explorations all suggest that $3 \mathrm{D}$ printing could be used to create a wide variety of pneumatic parts for interactive devices.

\section{D Printing vs. Alternative Fabrication Methods}

While some pneumatic controls could possibly be built with other methods like manual construction, casting or injection molding, 3D printing offers a unified, flexible approach to fabrication and customization. One of the key advantages of multi-material 3D printing, in particular, is precise automatic bonding of hard and elastic materials. For pneumatics, this is where air easily leaks and models break. Alternative techniques often bond elastic material to hard substrates, complicating construction. Methods include gluing [6], plastic welding, or coating hard parts with silicone to join them with other silicone segments [24], which are all time consuming and error prone. Furthermore, silicone coating is problematic for models that need low friction for rigid moving parts, such as the haptic knobs that we fabricate. On the flip side, the most significant downside to 3D printing, at present, is cost.

It is possible to fabricate some custom pneumatic devices by pouring silicone into a 3D printed mold $[1,10]$. The mold can be printed with flexible material to make it easier to remove, and its geometry can be specified using computer-aided design tools or can be automatically estimated from a target shape [16]. Electronic components can also be layered between silicone pours [18], including wire, conductive fabric, and magnetic Hall effect sensors. Modifying molds is very time consuming and prohibits rapid iteration, though.

Multi-material casting has also been demonstrated [20] and could potentially be used to create pneumatic parts with materials of different stiffness. Unfortunately, molds also limit castable forms. Some of our controls have interior geometries not possible with molds alone, requiring either multipiece molds (which are complicated) or consumable molds that are created and broken for every control. This significantly limits the scalability of these methods in comparison to multi-material 3D printing.

\section{Other Interactive Pneumatic Components}

Pneumatic systems have become popular for creating Organic User Interfaces [21], which consider shape as a key variable of interactivity. One way of creating these interfaces is by particle jamming $[5,19]$, where a jammable granular material is inserted into a flexible sack. The granular material slides around in a liquid-like state when loosely packed, but hardens into custom shapes due to inter-particle friction when the sack is vacuumed. Ou et al. [11] extended the above line of work by proposing layer jamming for thin sheet interfaces. A combination of copper tape and $3 \mathrm{M}$ Velostat ${ }^{\mathrm{TM}}$ can be layered into these structures to measure pressure and detect deformations. Air bladders can also be used as actuation mechanisms.

Inspired by advances in soft robotics, Yao et al. [24] created pneumatic soft composites for shape-changing interfaces, which can be fabricated in laboratories, e.g., using soft lithography. Their composites can significantly alter their shape by bending, curling, or expanding, based on the properties of the underlying layered materials, such as paper or fabric. Structural changes of these interfaces can be sensed with flexible circuitry made of copper tape, fluid conductors, or inkjet conductive ink. [24] also shows how a miniature pneumatic system can be built with off-the-shelf components for their practical applications. Our pressure control system is inspired by the latter. 


\section{Haptic Feedback}

The haptic sensations that we explore in this work were inspired by the interactive stylus of Lee et al. [8]. This pen generates a wide range of haptic feedback using a solenoid actuator, and has a sensitive tip to captures users' press force. In this manner, the pen can create the illusion of pressing buttons of several stiffness levels or can feel similar to pressing a two-level shutter button in a camera.

\section{D PRINTED VARIABLE-ACTIVATION FORCE CONTROLS}

The 3D printed device controls that we explore in this work rely on changes in air pressure to alter their physical feel and behavior. These controls can provide dynamic feedback during their manipulation. For example, variable activation force knobs can change physical resistance to turning actions based on a computational state (Fig. 1B). This enables richer interactions than equivalent static control devices.

Our interactive controls achieve a tight coupling of haptic feedback and functionality based on well understood pneumatic principles. This makes them easy to control, safe and inexpensive. In addition, pneumatics allow the location where force is applied to be decoupled from actuation, which facilitates the fabrication of small devices for physicallyconstrained user interfaces. These properties make pneumatic devices complementary to mechanical controls.

We used an Objet Eden $260 \mathrm{~V}$ printer with a resolution of 600 dpi (42 microns) to fabricate our controls. All models were 3D printed with VeroClear (rigid material) and TangoPlus (elastic material). ${ }^{1}$ Some models were then finished with acrylic paint. While current $3 \mathrm{D}$ printing materials may limit the performance of some pneumatic components (e.g., TangoPlus cannot elongate as much as Ecoflex $囚$ silicone), we expect better materials to become available in the future.

Our design considerations for these variable-activation force controls are presented in the following section, followed by our fabrication approaches. The rest of the paper is devoted to illustrative applications, as well as to the discussion of other aspects of the technology and its limitations.

\section{Design}

In general, we start our design process thinking about a desired haptic interface, its functionality and general shape. For example, we may want an interface with a haptic slider to provide input for a video game (as described in the Applications section). Then, we decide how we are going to sense user input with this device and how to incorporate air chambers with elastic parts into the geometry, such that the device can provide variable feedback. The interplay between the chosen sensing mechanism, and the shape and placement of the air chambers is key to obtaining the desired result.

\section{Sensing User Input}

We sense user interaction with pressure sensors, as well as with electrical components that operate based on connectivity and resistivity. In the first case, we attach differential pressure sensors to the pneumatic systems of the proposed physical

\footnotetext{
${ }^{1} \mathrm{~A}$ more detailed description of these materials can be found in http://www.stratasys.com/materials/
}

interfaces. For illustrative purposes, consider the conceptual diagram of Fig. 2, which we built for our applications. This system is composed of a pressure source (small compressor), two solenoid valves, a pressure sensor, and a 3D printed control with an air chamber. The valves are configured so that air can be added or released from the chamber under computer control, while the sensor continuously reads air pressure within the system allowing for precise, closed-loop pressure regulation. When the valves are at rest, as in the picture, the sensor allows us to detect when users alter the volume of the air chamber, e.g., by pressing a haptic button (Fig. 1A).

In the case of electrical conductivity and resistivity, we incorporate off-the-shelf sensors into our pneumatic controls once printed. The type of sensor used depends on the desired functionality of the control. For example, we use potentiometers to get users' input in the case of knobs and sliders, but could also incorporate off-the-shelf switches to our pneumatic buttons. Since these sensors are placed inside of our control devices, they generally constrain the shape and the design of the air chambers that we use to provide haptic feedback.

Figure 3 shows two off-the-shelf potentiometers that we incorporated into our haptic physical interfaces. When these components are added to the printed models, one can think of our pneumatic controls as an approach to augment the capabilities of standard input sensors.

Conductive materials will be widely available in the future for 3D printing, making it possible to print conductive paths for electricity to flow inside of pneumatic components [9]. Although, this technology is still being researched, we simulate this result by embedding thin layers of conductive material into our printed models, such as copper tape. This is an alternative to embedding simple off-the-shelf components while printing, which provides more design freedom.

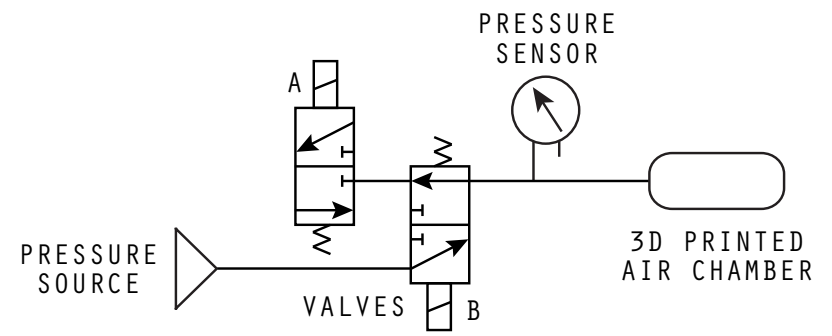

Figure 2. Prototype pneumatic system for some of our controls. The chamber is embedded in the controls and is used to provide feedback. When valves $A$ and $B$ are in their rest state, as in the picture, the system is closed and air cannot escape. When the valve $A$ is actuated and $B$ is at rest, the system is open and air is released. When $B$ is actuated but not $A$, a compressor (pressure source) increases air pressure in the system.

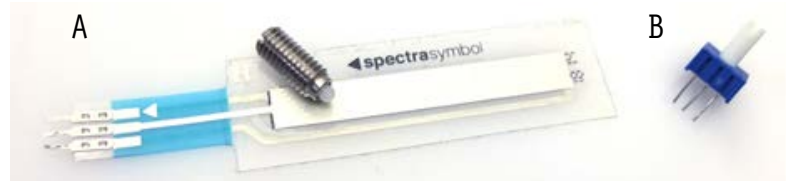

Figure 3. Off-the-shelf potentiometers used in some of our controls: SpectraSymbol's linear sensor and wiper (A), and rotary sensor (B). 
Variable Activation Force Capabilities through Air Chambers Air tight chambers are a key element of our variable activation force controls. These chambers are compressed air reservoirs, which we use to provide variable haptic feedback.

The chambers are made with materials of several elastomer characteristics and have at least one small, open orifice for air to flow through. Hard materials are typically used to provide chambers and their controls with a consistent, well defined shape. Soft materials are essential for the elastic parts of the geometry, which deform to provide variable haptic feedback or alter the physical form of a device.

Figure 4 illustrates several air chambers that can be 3D printed. Their elastic parts expand outwards from the chamber when internal pressure increases, generating forces perpendicular to their surface. In some cases, we use this force to resist pushing and squishing of the flexible parts, such as when users press a button. In others, we use this force to generate friction between some parts of our controls. As more friction is generated, our controls provide more resistance to user's actions (e.g., knobs become harder to turn).

We can increase pressure inside the chambers in several ways in order to produce different haptic feedback. Figure 5 shows some haptic waveforms that we can reproduce with our pneumatic system (Fig. 2). We open or close the valves to generate the different dynamic pressure profiles, keeping the compressor on continuously. In Fig. 5A, we repeatedly increase, hold, reduce, and hold the internal pressure of the system. In Fig. $5 \mathrm{~B}$, we increase pressure more slowly over time, release air from the system, and repeat. In the bottom cases we repeatedly increase pressure by steps (Fig. 5C) or linearly (Fig. $5 \mathrm{D})$, and then reduce pressure back to its original level.

The shape of the elastic part of the chambers affects their deformation and how much pressure they can hold. Printed chambers with round elastic parts, such as the one in Fig. 6, tend to be very robust. This chamber can hold up to about 7.25 psi $(50 \mathrm{kPa})$ before breaking. Other printed geometries have significantly lower pressure limits, such as the starshaped chamber of Fig. 1A. As expected, the flexible parts of the chambers tend to break in places where they connect to rigid material, and where their shape undergoes major deformations as pressure increases.

In general, we considered two types of forces during our design process. On one hand, there are internal forces, which we generate and control through the pneumatic system that drives our haptic controls. On the other, there are external forces that are present in the environment and are independent of the pneumatic system, such as gravity. The design considerations that we had with respect to the effect of these forces are further detailed the following sections, depending on the particular functionality of each control.

\section{Fabrication}

We fabricate pneumatic, variable-activation force controls using modern multi-material, photo-polymer 3D printing technology. This fabrication process is straightforward, replicable, and affordable when prototyping custom devices.
A
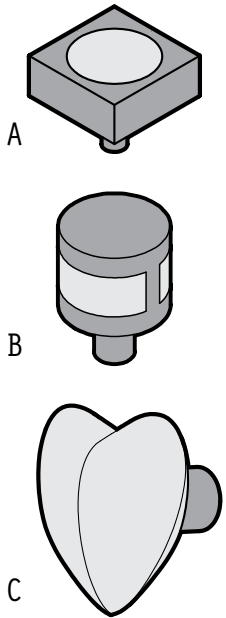
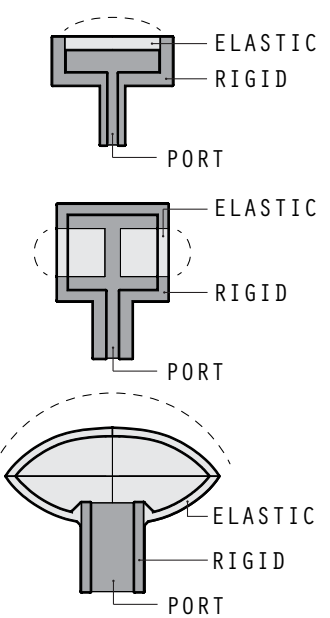

Figure 4. Several air chambers in their rest state (left) and a cross section that shows the internal flow of air (right). Air can come in or out of the chambers through the port at the bottom of each cross section. Parts made of elastic materials are colored in light gray, while rigid parts are darker. The dashed lines depict the deformation of the flexible parts when air is supplied through the ports, thus increasing internal air pressure. Our push buttons have chambers similar to (A), while our knobs have chambers like (B). 3D printing also offers the possibility of fabricating controls with more irregularly-shaped chambers, such as $(\mathrm{C})$.

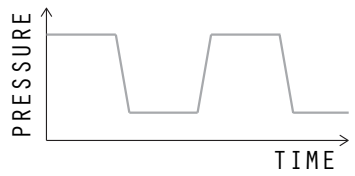

A

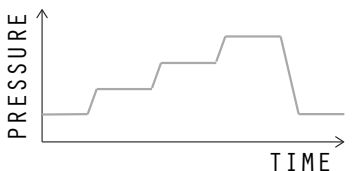

C

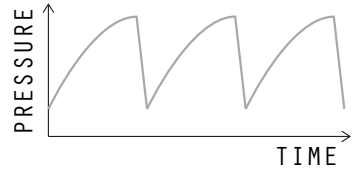

B

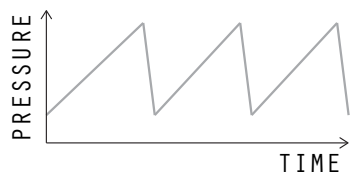

D
Figure 5. Dynamic pressure profiles that we can generate programmatically and render with our pneumatic controls.

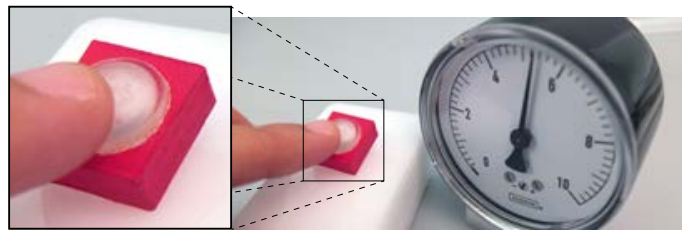

Figure 6. Printed pneumatic button (red) with an air chambers similar to Figure 4A. The button is placed on a stand (white) and is shown partially inflated, with a pressure of $5.4 \mathrm{psi}(37.2 \mathrm{kPa})$ relative to atmospheric pressure. When the button is fully inflated at $6 \mathrm{psi}(41.4 \mathrm{kPa})$, its top surface raises $3.5 \mathrm{~mm}$ in comparison to its position at 0 psi. The clear and elastic top of the button has a thickness of $1 \mathrm{~mm}$. 
The printing process starts with a digital geometric model of a control, which is then converted to slices, or layers, that are printed sequentially. Besides model materials, the printer can deposit a gel-like support material used to hold up overhanging geometry. This support material must be removed from the model after printing, e.g., with a waterjet cleaning unit or by manually rubbing it off from other materials.

Recent 3D printing technologies promise a future where all sorts of geometries for pneumatic devices and their chambers can be printed at once, immediately ready for use [3, 9]. Currently, however, these technologies are in their early stages and are not widely available. Instead, many commercial printers (like the one we use) rely on added support material to build models layer by layer.

Unfortunately, removing the support material from a printed model can be very difficult, especially when this material is inside small and tight geometries (such as tubes). Further, removing support material from soft surfaces can tear them. This is exactly what happens if we print our air chambers naively. Even after carefully trying to clean them, their interior is dirty with left-over support and their elastic parts are often broken as a result of the cleaning process. While remaining support material next to the rigid parts of the chambers does not pose much difficulty for their functionality, any support that remains next to the interior of the elastic parts can significantly affect haptic feedback since the support material is not very flexible. Another alternative to ease the cleaning process is to submerge printed models into a caustic bath, which dissolves support material. However, this technique can be a problem for embedded electronic components and, in our experience, it rarely removed all support from the models before starting to dissolve the elastic parts.

The following sections describe the approaches that we took to overcome the support material removal problem. These approaches are not mutually exclusive and should be adopted based on the desired printed geometry.

\section{Fabricating Chambers by Parts}

One approach to fabricate chambers consists of splitting the models into individual parts that can be easily assembled together. The goal of the splits should be to make the interior of the chambers more accessible. This way, we can more readily remove support material from the models once printed.

While the parts of a chamber could be put together in many ways, we found that $3 \mathrm{D}$ printing rigid threaded joints and assembling them with Teflon thread seal tape (or plumber's tape) was a robust approach for fabricating air-tight chambers. Due to printing resolution limitations, we opted for joints with coarse threads, which are also less likely to cross thread than finer counterparts. For example, we used threads with a major diameter between 8.5 and $50 \mathrm{~mm}$, and a pitch between 1.5 and $2.75 \mathrm{~mm}$. Sample screw models can be found in online design communities such as Thingiverse, ${ }^{2}$ or from parts sellers such as McMaster-Carr, ${ }^{3}$ which eases the incorporation of threaded joints into model geometry.

\footnotetext{
${ }^{2}$ http://www.thingiverse.com

${ }^{3} \mathrm{http}: / /$ www.mcmaster.com
}

Figure 7 shows a mushroom-shaped air chamber that can be 3D printed in parts. The top part of the chamber is made of both elastic and rigid materials, while the bottom part is completely rigid. When the parts are not assembled, the split allows better access into the chamber in comparison to printing the whole mushroom at once. Note that the size of the major diameter of the screw is the main limiting factor for accessing the top part of the chamber. The bigger the diameter, the more easily we can access the interior of the top component. When the chamber is assembled with plumber's tape around the screw joint, air can only enter or leave the pneumatic component through the port at the bottom of the mushroom.

Since threaded joints are standard in pneumatic systems, we can also print parts that directly attach to industry-standard, pre-fabricated threaded pipes and fittings. For example, the top part of the chamber in Fig. 7 could potentially connect to a pipe that follows the National Pipe Thread standard, rather than connecting to the stem of the mushroom.

We could also split the heart-shaped chamber of Fig. 4C into two parts, like we did for the mushroom. In fact, we can split many sorts of chambers into parts with screw joints as long as the joints are rigid and sufficiently large to be printed with at least a few connecting threads. The former is important because flexible joints are likely to let air escape when pressure increases. The latter guarantees that there is enough contact surface to keep the parts in place once assembled.

\section{Fabricating Chambers with Auxiliary Support}

An alternative method to print air chambers dispenses with the standard gel-like support material in their interior and replaces it with auxiliary, non-printed supports. More specifically, we insert pre-made parts that provide support for further building as in [7, 23]. In addition, we use these parts to create air-tight hollow spaces for our chambers, which do not need to be cleaned after printing. As a result, pneumatic controls are less prone to breaking during fabrication. While this strategy imposes constraints on the geometry of the model, as will be explained later, it is very attractive for printing pneumatic controls with little manual labor.

We use layers of acrylic as auxiliary support in our prototypes, and never remove them from the models once printed. An illustrative air chamber with auxiliary support in presented in Fig. 8. The layers of acrylic that we use to hold up geometry are fabricated by laser-cutting acrylic sheets. The shape of these pieces is based on 2D shapes derived from the underlying 3D CAD model for the part, such that they tightly

A
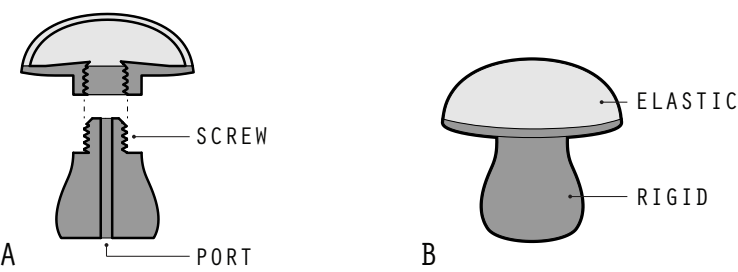

Figure 7. Cross section of the parts of a mushroom-shaped air chamber (A). During assembly, we add Teflon pipe tape to the screw of the bottom part, and insert this piece into the top one, resulting in (B). 
fit printed geometry. This is important since model material may sink if gaps exist between the layers of acrylic and the printed model. When this happens, the printed geometry may deform and undesired leaks may appear in the air chambers.

When we 3D print with auxiliary support, we disable printing with the standard gel-like support material, except for adding a pedestal at the bottom of the geometry - this is necessary to remove the the model from the printing tray once finished. We then start the printing process, and pause when a hollow space for the auxiliary support is layered (such as the "enclosure" in Fig. 8A). We place the auxiliary support material flush with the current top of the model and, finally, continue printing the model on top of the added component. In some cases, we may add more than one layer of auxiliary support to a printed control. For example, we added a second piece of acrylic to the model of Fig. 8 so that it could be printed with a barbed fitting. The barb is wider than the pipe that connects it to the model and, hence, needs to be supported.

We design the hollow spaces for auxiliary support material as part of our controls. This requires thinking about the orientation of the models as they are printed, such that the auxiliary support effectively holds up overhanging geometry. For example, the model of Fig. 8 has to be printed upside down to prevent the flexible part of the chamber to fall on top of auxiliary support. When single layers of acrylic are added to a model at a time, the height of the hollow spaces follows the thickness of the acrylic parts. Besides, model material is added all around auxiliary support when it has to be placed inside of an air-tight chamber. This ensures that air cannot freely escape the chambers other than through their ports.

While this fabrication approach is practical, it imposes an important constraint on the design of the models and the way they are printed: at any point in time during the printing process, the top of the model has to be flat (even when auxiliary support is added to printed geometry). This is required because we are printing layer by layer, and the print head needs to move close to the top of the model to deposit new material. As a consequence, all the layers of acrylic that we add to our controls lie parallel to the printing tray.

Another important consideration when fabricating chambers with this approach is that auxiliary support has to be rigid enough to hold up other geometry. Therefore, any elastic ma-
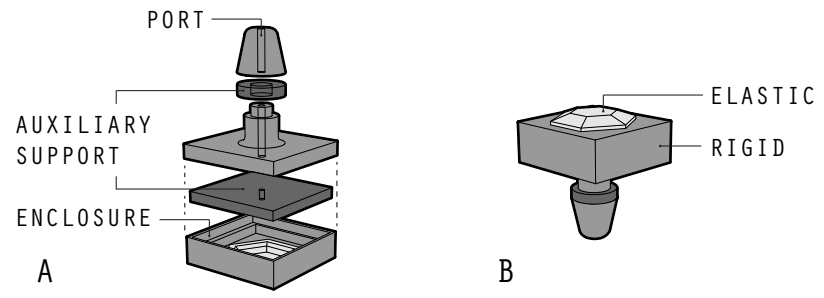

Figure 8. Auxiliary support for a pneumatic button. The model is displayed upside down in (A), as it would be $3 D$ printed. When the bottom enclosure is printed, the first piece of auxiliary support is inserted. When the next section is layered, auxiliary support in the form of a ring is added to the pipe of the chamber to hold up the rest of the model. No manual assembly is necessary after printing the complete model $(B)$. terial that is printed and cured on top of this support will likely lose flexibility, specially near the contact surface.

\section{Fabricating Custom Tools for Support Material Removal}

We can also 3D print custom tools to remove support material from the interior of our controls. These tools can be designed based on model geometry, such that they can easily reach support material in places that are otherwise hard to access. We used one of these tools to remove support material from the chamber of a pneumatic alligator toy, as shown in Fig. 9. The alligator is later described in our Applications section.

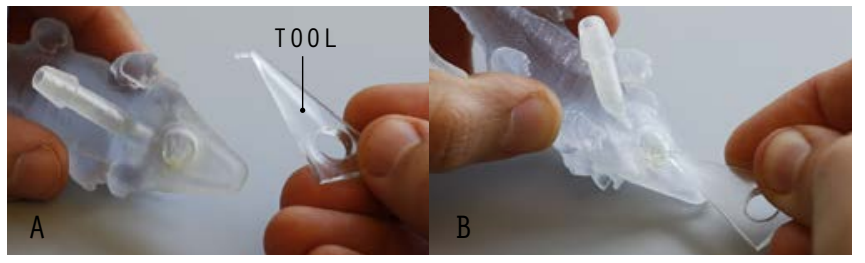

Figure 9. 3D printed tool (A) for removing support material (B)

\section{Other Fabrication Considerations}

We add 3D printed barbed connectors to all the air chambers that we fabricate, as in Figure 8. This standard connector prevents air from leaking through the ports of the chambers, and can be slightly reconfigured based on how we fabricate a model. For example, we typically make the internal diameter of the connector wider when we fabricate controls by parts, since support material has to be removed from their interior.

If a small and light component is embedded into a control while it is printed, it is likely that the head of the printer will move the component out of place when it slides on top of the model to deposit additional model material. In these cases, we add a few drops of a fast-acting cyanoacrylate-based adhesive (e.g., superglue) to the contact surface between the component and the part of the model that has already been printed. This extra step ensures that the component is held securely in place during the printing process.

Note that the constraints that limit the placement of layers of acrylic into our models while printing also apply to other embedded parts, including electronics. An example of embedded copper tape is presented in the next section.

\section{Device Controls}

We categorize the types of controls that we fabricate based on the type of actions that users perform to provide input:

\section{Pressing and Squishing}

We fabricate controls that can be pressed (like a standard button) and others that can be more roughly "squished". The main differences between these types of controls are their size and the roundness of their elastic parts. The bigger and rounder the elastic part of a chamber, the more "squishy".

Figure 10 shows star-shaped pneumatic buttons. User input can be sensed with a pressure sensor attached to the pneumatic system that drives the mechanisms. The curve in the background of Fig. 1A shows variations in the internal pressure of the big star as it is pressed. Note that we can scale a 
pneumatic control, such as in Fig. 10A, and test it immediately by simply adjusting the pressure range at which it operates. This is an advantage of pneumatics for prototyping device controls in comparison to mechanical counterparts.

We can also incorporate electronics into pneumatic buttons to sense when users fully depress their elastic top, as in Fig. 11. This allows the input to be received irrespective of pressure changes, which is useful when we provide continuous, variable haptic feedback. The button in Fig. 11 was printed with auxiliary support, and with an extra layer of acrylic that holds two thin stripes of copper tape. Additional copper tape was placed on the interior surface of the elastic part, such that it closes the circuit when it is fully depressed. As with the model of Figure 8, this button had to be printed upside down.

Furthermore, we can use the deformation of buttons to create actuated switches. To this end, we incorporated the round button of Fig. 6 into a structure with a hinge joint (Fig. 12). When pressure increases, the overhanging part of the switch raises; when pressure decreases, it lowers down due to gravity. Pneumatic controls could potentially be incorporated into more complex structures with other printed joints [2].

\section{Turning}

We fabricate knobs that resist users' turning actions with chambers similar to the one in Figure 4B. We add potentiometers inside of these controls to sense the orientation of their caps and when when users rotate them.

Figure 13 shows an illustrative knob that can provide variable feedback no matter in which direction it is turned. We printed the base (with the air chamber) independently of the cap. Even though the round shape of the chamber could very well hold a screw joint, we printed it with auxiliary support such that we did not have to remove support material from its interior. We inserted a potentiometer into the base during assembly and attached the cap to this sensor as a press-fit component. We made the elastic parts of the potentiometer slightly thicker than for the other models $(1.5 \mathrm{~mm}$ vs $1 \mathrm{~mm})$ since it alters the rigidity of the knob based on friction. This elastic part can still deform, though, as shown in Fig. 13C. With this deformation, we can provide at least three levels of feedback with pressures from 0 to $3.6 \mathrm{psi}(24.8 \mathrm{kPa})$.

We can also provide different feedback based on the direction in which a knob is turning. We accomplish this by adding a sawtooth pattern to the internal walls of the cap and its elastic parts (Figure 14). Counter-clockwise turns are easier than clockwise rotations with this pattern.

\section{Sliding}

In addition, we can fabricate sliders that provide variable resistance to users' actions. We offer an example mechanism in Figure 15A. This model was printed in three parts: the base chamber, the rails, and the handle; and was then assembled as shown in the picture. While we could have added an off-theshelf slider to this device control, we opted for incorporating a linear membrane potentiometer to the model instead. This allowed us to customize the shape of the handle, showing the versatility of $3 \mathrm{D}$ printing.

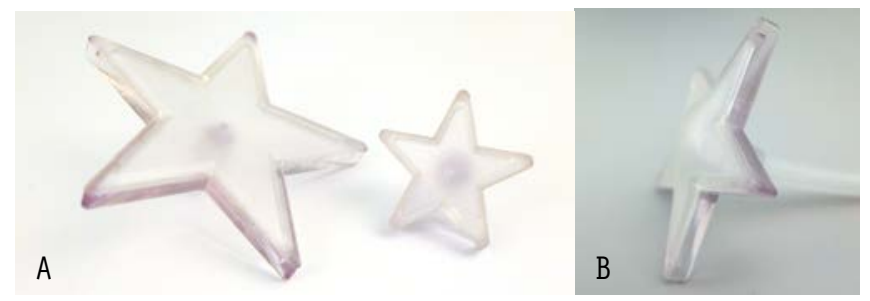

Figure 10. Star buttons fabricated with auxiliary support (A). The big star is inflated in (B) at $0.6 \mathrm{psi}(4.1 \mathrm{kPa})$ relative to atmospheric pressure.
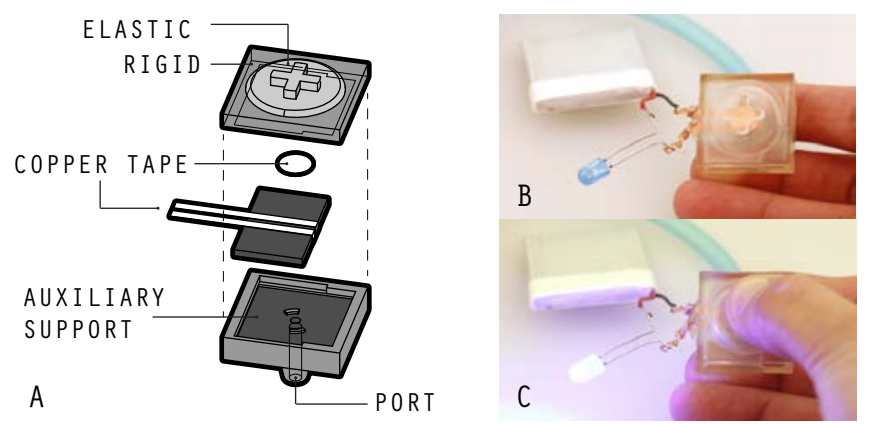

Figure 11. Pneumatic button that was printed with auxiliary support and with an internal conductive circuit made of copper tape. (A) shows the placement of copper inside the printed model, (B) shows the button in its rest state, and (C) shows the button inflated and pressed. The round piece of copper attaches to the internal bottom surface of the elastic part, and makes contact with the two copper stripes when the button is pressed. This button was printed upside down, as the one in Fig. 8.

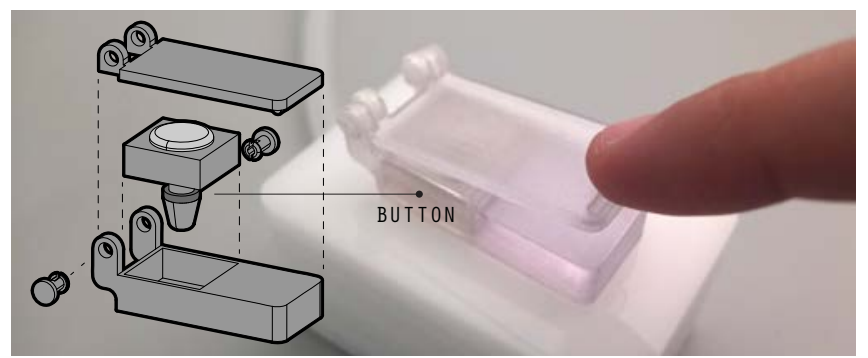

Figure 12. 3D printed switch. We inserted a button like the one in Fig. 8 into a printed structure that was fabricated by parts and assembled as in the illustration. When the button is deflated, it cannot be pressed since the rigid top of the switch is in contact with the bottom. However, when the button is inflated, the top part of the switch raises. We can then detect users' presses with a pressure sensor.
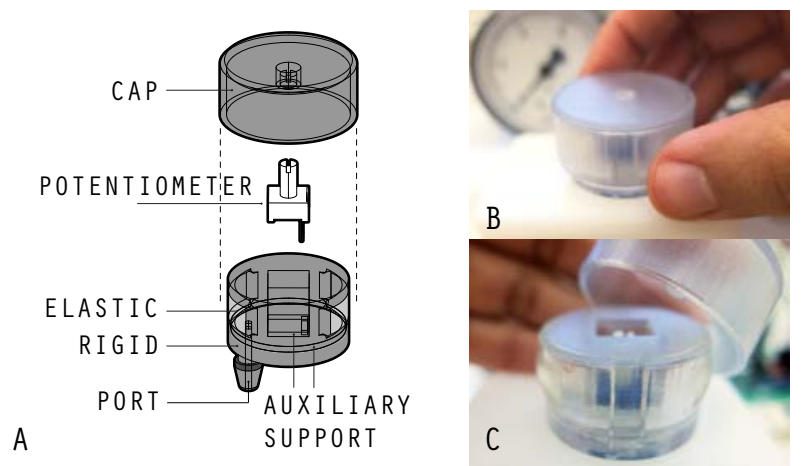

Figure 13. 3D printed knob with a chamber as in Figure 4B. (A) shows the parts that make the knob and how its assembled after printing. (B) shows the resulting component, and (C) shows the internal chamber inflated at $3.6 \mathrm{psi}(24.8 \mathrm{KPa})$ with respect to atmospheric pressure. 

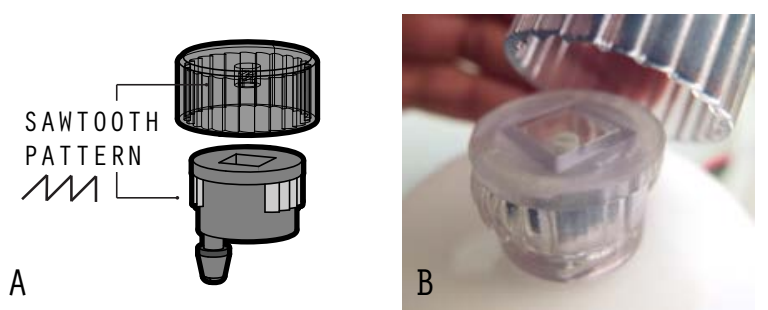

Figure 14. Sawtooth knob. The elastic walls of the chamber and the internal surface of the cap have a sawtooth pattern (A). This allows to provide different feedback based on which orientation the cap rotates. (B) shows the air chamber of the knob in its inflated state, when it can provide resistance to turning actions.

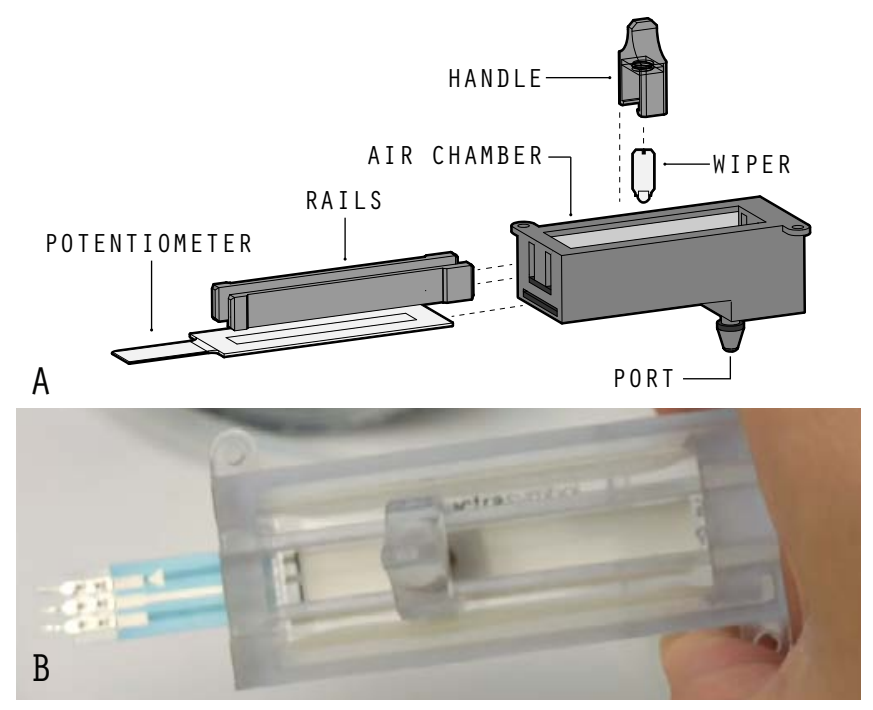

Figure 15. 3D printed slider with the linear potentiometer and wiper of Fig. 3. The base chamber was fabricated with auxiliary support independently of all other parts (A). During assembly, we screw the wiper into the handle, slide the potentiometer and rails into the base, and snap the handle onto the rails. When the elastic walls of the chamber inflate, they make pressure against the handle (B), making the slider stiffer.

The slider that we printed also locks in place - users have to push down on the handle in order to move it. We accomplished this feature by screwing a wiper with an embedded spring into the handle. This wiper also allows for precise sensing with the linear potentiometer. When air pressure increases inside the chamber, its elastic parts inflate inwards towards the handle (Fig. 15B). This generates friction that resists the linear motion of the slider. As with the knob, we were able to create three distinguishable levels of feedback at $0 \mathrm{psi}, 0.6 \mathrm{psi}(4.1 \mathrm{kPa})$, and $0.8 \mathrm{psi}(5.5 \mathrm{kPa})$.

\section{Other Device Controls}

While our work spans the design space of the controls that are most often used in physical control panels, we foresee other types of pneumatic controls being fabricated akin to our prototypes. For example, one could 3D print a pulling control with a design similar to the chamber in Fig. 4B. Imagine replacing the hard material in the middle of the chamber with elastic material and adding a small inward curvature to the elastic walls all around. Then, it would be possible for users to provide input by pulling the top of the chamber up- wards, and we would be able to provide feedback by varying pressure inside the chamber. If pressure increases, the top of the chamber would move up, thus decreasing the range of pulling motion available. Another example are bending controls. Consider the bending building block of [17] for prototyping manipulation affordances on robot skins. If one prints a chamber with that shape, make it air-tight, and regulate pressure inside of it, then the chamber could be used for providing both user input and haptic feedback. As pressure increases, this control would become harder to bend.

\section{APPLICATIONS}

We fabricated a set of interactive devices to illustrate potential applications for 3D printed variable activation force controls. For all of the examples that follow, we used a pneumatic configuration similar to that of Fig. 2. The specific electronic and pneumatic components that we used are depicted in Fig. 16.

\section{Alligator Toy}

We used the same operating principles of the switch of Figure 12 to make a $3 \mathrm{D}$ printed alligator toy. Rather than building the device with auxiliary support, we fabricated it in parts (Figure 17A). This allowed us to route air down through the alligator belly, where it is hidden from users. The elastic part of the chamber in the lower jaw of the alligator pushes the top of its head upwards when pressure increases. As a result, it looks like the alligator opens its mouth. When pressure is reduced, the top of the head falls to its rest position due to gravity. If the internal pressure of the alligator is at least $4 \mathrm{psi}(27.6 \mathrm{kPa}$; when its mouth is slightly open), then we can sense when users press on its nose using a pressure sensor. In response, we make the alligator open and close its mouth repeatedly, e.g., with a square haptic waveform as in Figure 5A.

\section{Responsive Lamp}

We made an interactive lamp by incorporating three light emitting diodes (LEDs) into a 3D printed pneumatic device. Figure 18A shows the components of the lamp, and Figures $18 \mathrm{~B}$ and $18 \mathrm{C}$ show the lamp slightly inflated and being pressed, respectively. Our lamp can respond to a variety of input gestures measured by small pressure changes. For example, long and short squeezes change its brightness, while hue is modified based on squeeze strength and duration. Haptic feedback is utilized to indicate important changes in the internal computational state of the lamp.

\section{Volume Knob}

We used the sawtooth knob from Fig. 14 to provide haptic volume control, as depicted in Fig. 19. Clockwise and counter-clockwise rotations increase and lower volume, respectively. In order to keep volume levels in a safe range, our prototype application increases pressure as the knob is rotated clockwise beyond its middle position. This alerts users that they are close to reaching unsafe volume levels. When the maximum safe volume level is reached, the chamber fully inflates, providing maximum resistance to additional clockwise rotations. Lowering the volume is still easy, though.

\section{Side Scrolling Video Game}

We used the slider from Figure 15 to provide input and haptic feedback for a side scrolling video game. In this game, users 


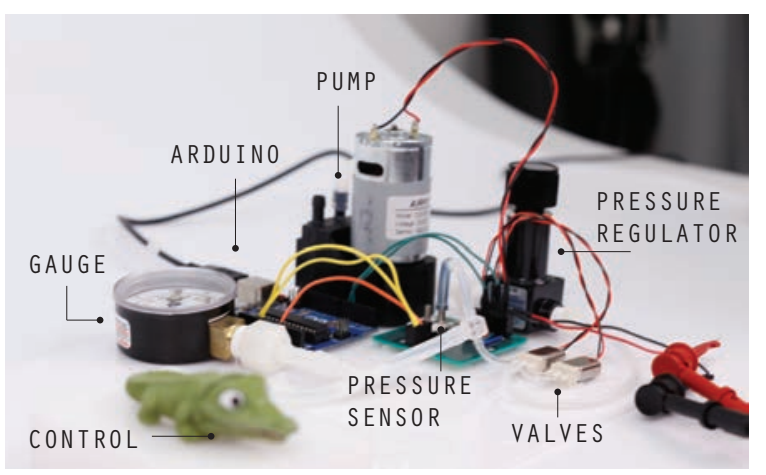

Figure 16. Pneumatic and electronic system used for all applications, which includes an AIRPO pump (model D20288), a manual pressure regulator by Airtrol, two SMC valves (model S070C), a Silicon Microstructures pressure sensor (model SM5651), a gauge, and an Arduino. The pressure regulator and the gauge are not necessary for operating our controls, but were added for safety.
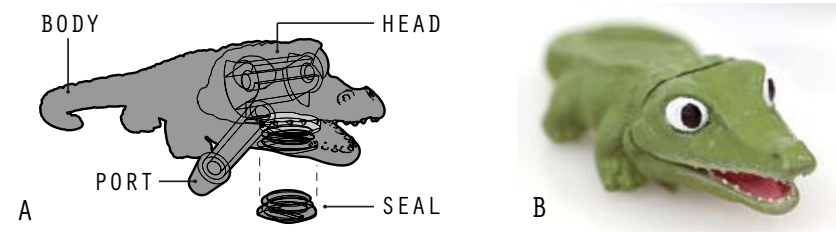

Figure 17. Alligator toy. The body, head and seal were printed independently and then assembled (A). The head snaps into the body and the seal screws into a hole in the bottom of its jaw. The elastic part on the mouth (lighter gray) inflates when pressure increases, opening the mouth (B). Fig. 9 shows the unfinished body part after printing.
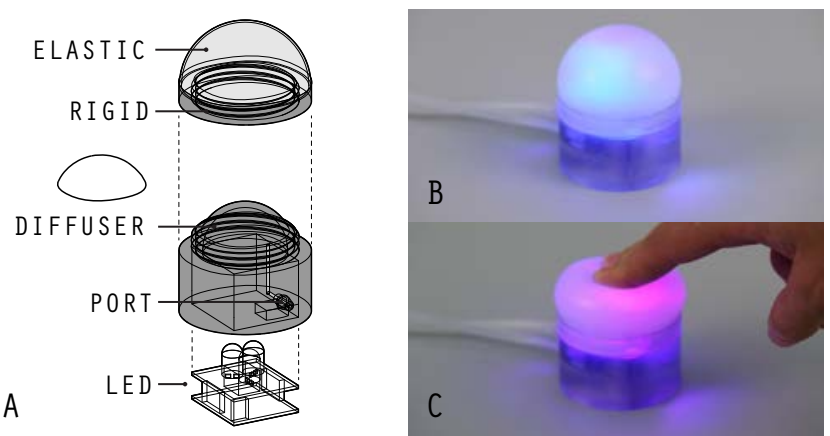

Figure 18. Pneumatic lamp. The device was printed by parts and assembled (A). We added a diffuser to the base and inserted an LED inside. (B) shows the lamp slightly inflated, and (C) shows it being pressed.

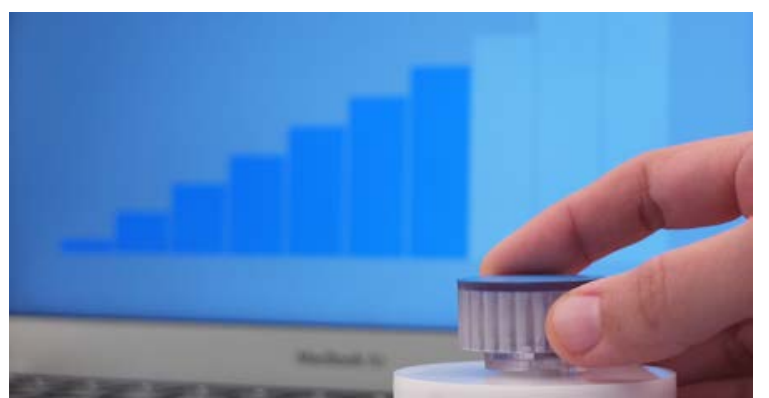

Figure 19. We use the sawtooth knob of Figure 14 to control volume in a prototype application. The current volume level is rendered real-time on the screen (as in the background of the image). control the vertical position of an airplane as it flies through a cloudy sky (Figure 20). The slider sets the vertical velocity of the plane; in the middle position, the airplane has zero vertical velocity and simply moves horizontally. When the slider is adjusted up or down, it controls the plane's upward or downward movement in a rate controlled manner.

When the plane is flying through clear sky, the slider is set at its minimum resistance level $(0 \mathrm{psi})$. To simulate little turbulence, the slider becomes harder to move when flying through smaller clouds $(0.6 \mathrm{psi}, 4.1 \mathrm{kPa})$. When the plane passes through the biggest clouds in the game, we increase the pressure of the slider to $0.8 \mathrm{psi}(5.5 \mathrm{kPa}$; as in Fig. 15B). The slider can still be moved, but with more difficulty.

\section{Other Applications}

Other applications could benefit from variable activation force controls. For example, alarm clocks could use a pneumatic snooze button to highlight the state of the alarm, and prevent accidental presses that may shut down the alarm unintentionally. Radio tuners could also use pneumatic knobs to indicate when users reach a station. This becomes specially useful when users have to pay attention to other tasks, e.g., when they are driving and looking for a particular station. Finally, we also envision printed pneumatic components as fully-customized building blocks of free-form hardware interfaces, such as Palette. ${ }^{4}$

\section{FUTURE WORK}

We designed our pneumatic controls manually, based on the desired haptic feedback and sensing modality. However, this process could be semi-automated based on a set of predesigned air chambers. Making an interface to facilitate nonexpert design of these controls is an important area of future work. Likewise, it would be useful to provide a computational interface for the rapid design of 3D printed tools for support material removal. This could potentially ease, even further, the fabrication of pneumatic controls.

We used acrylic as auxiliary support, but foresee other materials being used as well to hold up printed geometries. For example, one could think of 3D printing a part, e.g., an air

\footnotetext{
${ }^{4}$ http://palettegear.com
}

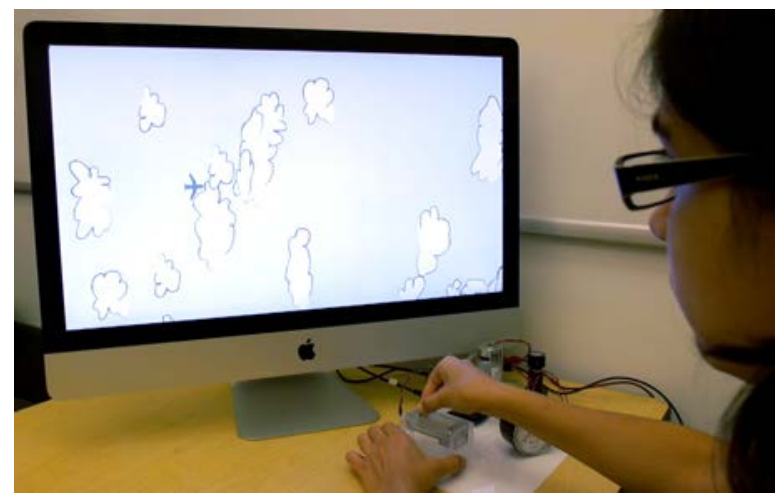

Figure 20. Flying game with pneumatic slider. The slider provides input vertical velocity for the plane as it flies through the clouds. When the plane hits turbulence, the slider becomes stiffer. 
chamber, and then embedding it into another model while it is printing. This could potentially reduce the time and effort put into manually assembling printed controls, and may as well allow to create more complex air chambers.

Our work focused on the design and fabrication of devices that operate with air. While many considerations were made based on the physical properties of this compressible gas, we believe that our design rationale and fabrication approaches can guide the rapid prototyping of control devices that use other fluids, such as water. This could offer faster and less compliant feedback, which may benefit certain applications.

\section{ACKNOWLEDGMENTS}

We are thankful to Disney Research and The Walt Disney Corporation for continued support of this research effort. Thanks to Moshe Mahler for modeling the alligator.

\section{REFERENCES}

1. Borgatti, M. Thingiverse: Trefoil tentacle. http: //www.thingiverse.com/thing : 92103. Last accessed on $08 / 27 / 14$.

2. Calì, J., Calian, D. A., Amati, C., Kleinberger, R., Steed, A., Kautz, J., and Weyrich, T. 3d-printing of non-assembly, articulated models. ACM Trans. Graph. 31, 6 (Nov. 2012).

3. Clark, L. How hair gel enables freeform $3 \mathrm{~d}$ printing with an undo function. Wired.co.uk, July 2013.

http: //www.wired.co.uk/news/archive/2013-07/25/ undo-3d-printing.

4. Clarkson, R. Blossom. http: //www . richardclarkson. com/blossom. Last accessed on 01/02/15.

5. Follmer, S., Leithinger, D., Olwal, A., Cheng, N., and Ishii, H. Jamming user interfaces: Programmable particle stiffness and sensing for malleable and shape-changing devices. In Proc. UIST'12 (2012).

6. Harrison, C., and Hudson, S. E. Providing dynamically changeable physical buttons on a visual display. In Proc. CHI'09 (2009), 299-308.

7. Kataria, A., and Rosen, D. W. Building around inserts: methods for fabricating complex devices in stereolithography. Rapid Prototyping Journal 7, 5 (2001), 253-262.

8. Lee, J. C., Dietz, P. H., Leigh, D., Yerazunis, W. S., and Hudson, S. E. Haptic pen: A tactile feedback stylus for touch screens. In Proc. UIST'04 (2004), 291-294.

9. Malone, E., and Lipson, H. Multi-material freeform fabrication of active systems. In Proc. ESDA'08 (2008).

10. Mosadegh, B., Polygerinos, P., Keplinger, C., Wennstedt, S., Shepherd, R. F., Gupta, U., Shim, J.,
Bertoldi, K., Walsh, C. J., and Whitesides, G. M. Soft robotics: Pneumatic networks for soft robotics that actuate rapidly. Advanced Functional Materials 24, 2109 (2014)

11. Ou, J., Yao, L., Tauber, D., Steimle, J., Niiyama, R., and Ishii, H. jamsheets: Thin interfaces with tunable stiffness enabled by layer jamming. In Proc. TEI'14 (2014).

12. Robins, M., and Bean, H. Variable force camera control, Oct. 4 2005. US Patent 6952528.

13. Russell, L. 3d printed pneumatic gripper. http:// getitmade.com.au/3d-Printed-Pneumatic-Gripper. Last accessed on 08/27/14.

14. Savage, V., Schmidt, R., Grossman, T., Fitzmaurice, G., and Hartmann, B. A series of tubes: Adding interactivity to $3 \mathrm{~d}$ prints using internal pipes. In Proc. UIST'14 (2014).

15. Shkolnikov, V., Ramunas, J., and Santiago, J. G. A self-priming, roller-free, miniature, peristaltic pump operable with a single, reciprocating actuator. Sensors and Actuators A: Physical 160, 1 (2010), 141-146.

16. Skouras, M., Thomaszewski, B., Bickel, B., and Gross, M. Computational design of rubber balloons. Proc. Eurographics (2012).

17. Slyper, R., and Hodgins, J. Prototyping robot appearance, movement, and interactions using flexible $3 \mathrm{~d}$ printing and air pressure sensors. In Proc. RO-MAN'12 (2012).

18. Slyper, R., Poupyrev, I., and Hodgins, J. Sensing through structure: Designing soft silicone sensors. In Proc. TEI'11, ACM (New York, NY, USA, 2011), 213-220.

19. Stanley, A., Gwilliam, J., and Okamura, A. Haptic jamming: A deformable geometry, variable stiffness tactile display using pneumatics and particle jamming. In World Haptics Conference (WHC) (April 2013).

20. Uribe, C. D. G. Multi-material Molding and Casting Mid-sole. Master's thesis, MIT Media Lab, 2014.

21. Vertegaal, R., and Poupyrev, I. Organic user interfaces. Communications of the ACM 51, 6 (2008), 26-30.

22. Walus, K. Thingiverse: Adjustable one way valve. http: //www.thingiverse.com/thing : 62728. Last accessed on $08 / 28 / 14$.

23. Willis, K., Brockmeyer, E., Hudson, S., and Poupyrev, I. Printed optics: $3 \mathrm{~d}$ printing of embedded optical elements for interactive devices. In Proc. UIST'12 (2012).

24. Yao, L., Niiyama, R., Ou, J., Follmer, S., Della Silva, C., and Ishii, H. Pneui: Pneumatically actuated soft composite materials for shape changing interfaces. In Proc. UIST'13 (2013), 13-22. 